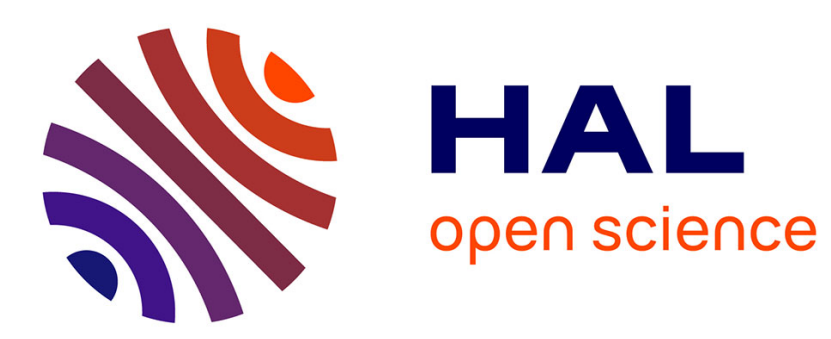

\title{
Directionality Measurement and Illumination Estimation of 3D Surface Textures by Using Mojette Transform
}

\author{
Peng Jia, Junyu Dong, Lin Qi, Florent Autrusseau
}

\section{To cite this version:}

Peng Jia, Junyu Dong, Lin Qi, Florent Autrusseau. Directionality Measurement and Illumination Estimation of 3D Surface Textures by Using Mojette Transform. 19th International Conference on Pattern Recognition, Dec 2008, Tampa, United States. pp.1144. hal-00324244

\section{HAL Id: hal-00324244 \\ https://hal.science/hal-00324244}

Submitted on 12 Jul 2010

HAL is a multi-disciplinary open access archive for the deposit and dissemination of scientific research documents, whether they are published or not. The documents may come from teaching and research institutions in France or abroad, or from public or private research centers.
L'archive ouverte pluridisciplinaire HAL, est destinée au dépôt et à la diffusion de documents scientifiques de niveau recherche, publiés ou non, émanant des établissements d'enseignement et de recherche français ou étrangers, des laboratoires publics ou privés. 


\section{Cover Page}

1) Title of the paper:

\section{Directionality Measurement and Illumination Estimation of 3D Surface Textures by Using Mojette Transform}

2) authors' affiliation and address:

IRCCyN-IVC, (UMR CNRS 6597), Polytech' Nantes

Rue Christian Pauc, La Chantrerie, 44306 NANTES, France.

Tel : 02.40.68.30.52

Fax : 02.40.68.32.32

3) e_mail address:

Florent.Autrusseau@univ-nantes.fr

4) Conference \& Publisher information:

IEEE ICPR 2008

http://www.icpr2008.org/

http://www.ieee.org/

5) bibtex entry:

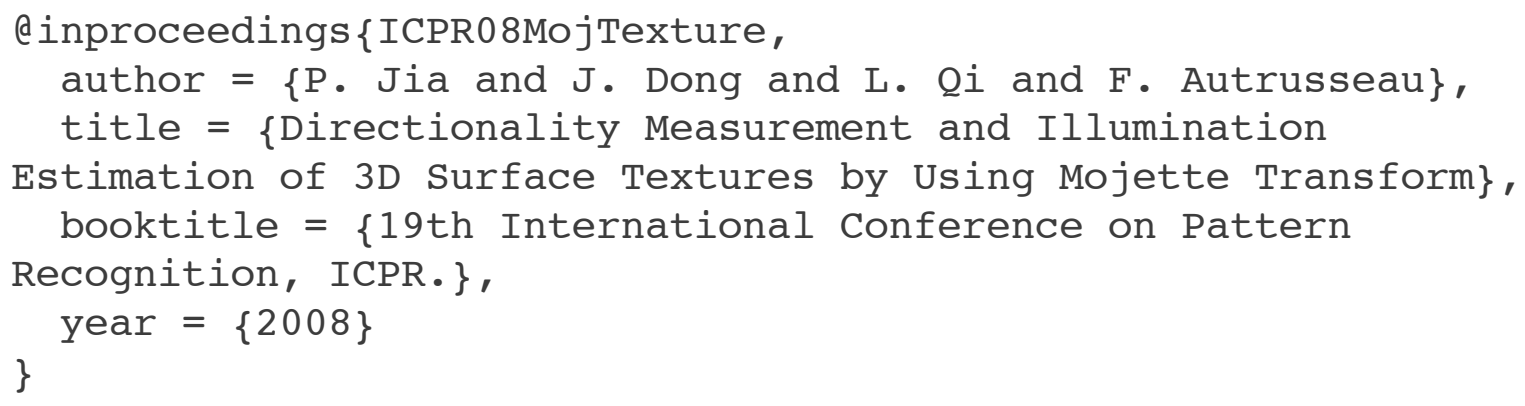




\title{
Directionality Measurement and Illumination Estimation of 3D Surface Textures by Using Mojette Transform
}

\author{
Peng Jia Junyu Dong Lin Qi \\ Computer Science Department, Ocean University of China \\ dongjunyu@ouc.edu.cn \\ Florent Autrusseau \\ IRCCyN-IVC, UMR CNRS 6597, Ecole polytechnique University of Nantes \\ Rue Christian Pauc, La Chantrerie, BP50609, 44306 NANTES, CEDEX 3 \\ Florent.Autrusseau@Polytech.univ-nantes.fr
}

\begin{abstract}
This paper presents a new approach to measure texture directions and estimate illumination tilt angle of $3 D$ surface textures by using mojette transform. Feature vectors are generated from variances of 72 mojette transform projections with different project angles. The measured texture directions are compared with human perceptional judgement. Further more, we estimate illumination tilt angles by minimizing the Euclidean distance of the feature vector between the test image and the training sets. Experimental results show the effectiveness and accuracy of our proposed approach.
\end{abstract}

\section{Introduction}

Texture analysis plays an important role in computer vision and computer graphics. Direction of texture image is a strong influence when people perceive a texture. Texture direction analysis is widely used to create rotation invariant classifiers[8][11][4]. And many direction measurement methods have been proposed[7][10], which are only involved in 2D texture images.

However, real-world textures are seldom "flat" and normally comprise rough surface geometry and various reflectance properties, which can produce dramatic effects on the appearance of the sample surfaces under varied illumination and viewing conditions[3]. Figure 1 shows two example images of a 3D surface texturea piece of wallpaper illuminated from two directions. The difference is obvious. This presents challenges in both computer vision and computer graphics. The directionality of the $3 \mathrm{D}$ surface may be weakened when given an illumination from a direction which is similar with the surface's intrinsic direction. This is illustrated in the right image in figure 1 , the vertical texture direction is remarkably weakened by the $90^{\circ}$ illumination. It is therefore important to capture the characteristics of 3D surface textures so that successive analysis can be achieved.

We investigates the directionality measurement of the 3D surface textures in this paper. Mojette transform, a discrete form of radon transform, is used to capture the directional information of surface images under different illumination conditions. Further more, when given a novel image of one surface under unknown illumination condition, we try to estimate the tilt illumination angle.
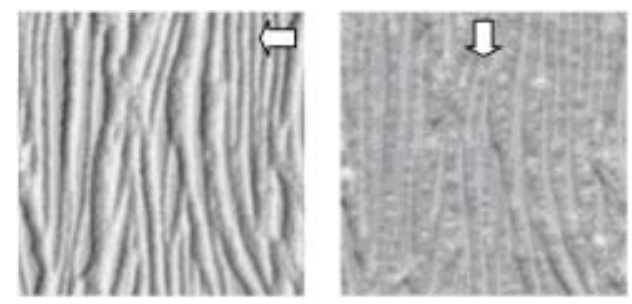

Figure 1. Two images of a 3D surface texture illuminated from different directions. The block arrows show the illuminate directions.

The outline of the rest of the paper is as follows: in section 2, we show our mojette transform manipulation on 3D surface textures. Next, section 3, we present our directionality measurement method. The illumination estimation is presented in section 4. And we conclude our work in Section 5. 


\section{Mojette transform on 3D surface textures}

The Mojette transform[2][6] is an exact discrete Radon transform [9] defined for specific projections angles $\tan \theta=q / p$ where $(p, q)$ are both integer restricted to $q>0, p \in Z$, or $q=0$ and $p=1$ and with $G C D(p, q)=1$. Each component of a projection is called a bin, the value of this latter being the sum of all the pixels crossed by the appropriate projection line. The transform is defined for each direction by the following $M_{p, q}$ operator :

$$
M_{p, q} f(k, l)=\sum_{k=-\infty}^{+\infty} \sum_{l=-\infty}^{+\infty} f(k, l) \Delta(b+q k-l p)
$$

Where $\Delta(b)=\left\{\begin{array}{l}1 \text { if } b=0 \\ 0 \text { if } b \neq 0\end{array}\right.$, and where the Mojette transform $M_{I} f(k, l)$ is described as the set of $I$ projections: $M_{I} f(k, l)=\left\{M_{p i, q i} f ; i \in 1,2 \cdots I\right\}$. The angle restriction (compared to classical Radon transform) leads both to a specific sampling on each projection and to a number of bins $(p, q)$ angle-dependent.

Mojette transform, as well as radon transform, is widely used to analyze images[2] or to estimate image(including texture) directions[8]. In [8], second derivative of variance of each projection is used to determine the texture direction, but this stands only when the texture has a significant directionality.

Cause directionality of texture is much more a concept in psychological domain, different people may perceive one same texture having different direction. This phenomena will be even worse on 3D surface textures, since the illumination acts as a direction filter[5]. We try to measure this problem in a quantitative analysis.

We exploit the PhoTex texture database (www.macs.hw.ac.uk/texturelab/database)[1] for experiments. The database contains many sample textures; each texture is represented using 36 images captured under a variety of different illumination conditions. The illumination angle is denoted by a slant (zenith) angle a tilt (azimuth) angle. There are three slant angles $\left(45^{\circ}, 60^{\circ}\right.$ and $\left.75^{\circ}\right)$. Under each slant angle, there are 12 tilt angles (from $0^{\circ}$ to $330^{\circ}$ with a step of $\left.30^{\circ}\right)$.

In our experiments, we only take the $N_{\text {tilt }}=12 \mathrm{im}$ ages of different tilt angles under slant angle of $45^{\circ}$ as the surface image set, because the change in slant angle introduces slight effect to the directionality of the texture. For each image in one surface set, we conduct $N_{\text {proj }}=72$ mojette transform projections with projection angle from $0^{\circ}$ to $180^{\circ}$. And then, we calculate the variance of each projection. This means a $N_{\text {proj-dimension column vector } V \text { is derived from each }}$ image. After applying this process to each image in one set, we can get a $N_{\text {proj }} \times N_{\text {tilt }}$ matrix for one surface image set, denoted as $V M \in \mathbb{R}^{N_{\text {proj }} \times N_{\text {tilt }}}$. This matrix is used to measure the directionality and estimate tilt illumination angle in the following sections. Figure 2 is an example of matrix $V M$ of surface "ace" which has a strong vertical directionality.

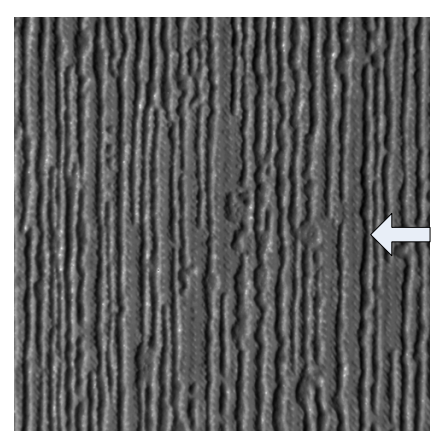

(a)

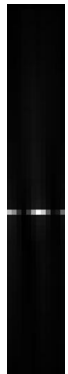

(b)

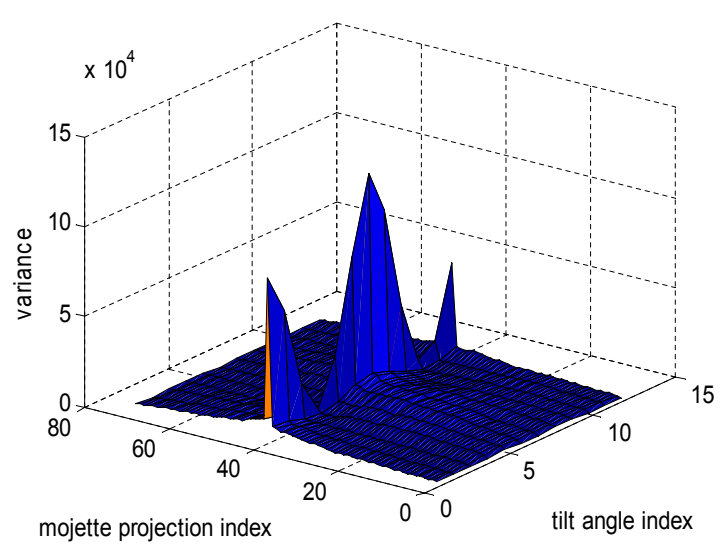

(c)

Figure 2. Mojette transform on one surface set. (a) is a sample image of surface "ace" with tilt angle of $0^{\circ}$. The block arrow indicates the tilt illuminate direction. (b) is the scaled image representation of $V M$ matrix derived from surface "ace" image set. (c) is the 3D plot of $V M$.

\section{Direction measurement}

As illustrated in previous section, we can not measure the direction of a 3D surface texture just by one single image. After calculating the matrix $V M$ of each 3D surface image set, we can analyze this matrix and draw some conclusions about how the directionality changes with projection directions and illumination directions. 
It is obvious that the variance value of the mojette projection peaks when the project direction is parallel with the texture direction and reaches lowest point when the project direction is orthogonal with the texture direction. This is the intrinsic character of mojette transform. Contrarily, the illumination acts the other way round. The principal directional geometric elements response strongly when given an orthogonal illumination. A straightforward method is derived to eliminate the influence that the illumination brings.

We sum up the matrix in each row to generate a

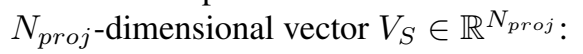

$$
V_{S i}=\sum_{j=1}^{N_{\text {tilt }}} V M_{i, j} \quad i=1 \cdots N_{\text {proj }}
$$

For analytical convenience, we scaled this vector by dividing each element by $\sum_{i=1}^{N_{\text {proj }}} V_{S}$, so that the sum of the vector elements equals to 1 .

By cumulating the $V M$ matrix in row, we can effectively eliminate illumination influence to the directionality. This is because that Like [8], we calculate the second derivative of $V_{S}$, denoted as $V_{S}^{\prime \prime}$. The principal surface texture direction is at where the $V_{S}^{\prime \prime}$ reaches its minimum value. Figure 3 gives an example of the $V_{S}$ and $V_{S}^{\prime \prime}$ of surface "ace" (sample image (a) in Figure 2).
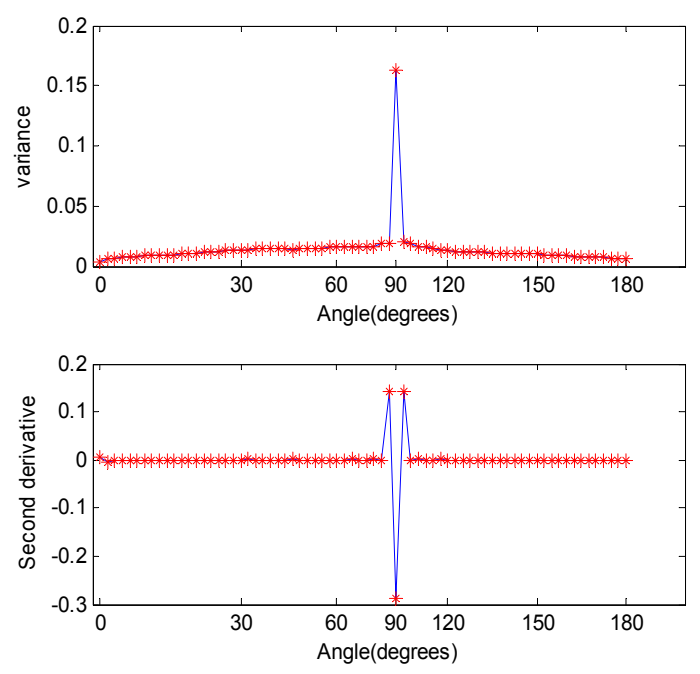

\section{Figure 3. Plots of $V_{S}$ (1st row) and $V_{S}^{\prime \prime}$ (2nd row) of surface "ace"}

Since only a limited number of surfaces have such strong directionality like "ace", some surfaces may have several direction or no significant direction (isotropic) from human's perception view. We proposed a measurement of the directionality of 3D surfaces. For each
$V_{S}^{\prime \prime}$, we set a threshold of 0.01 , and the elements below this threshold indicate the candidate directions of the surface. The following are discussion about the number of points below the threshold:

1.No elements. This means the surface is isotropic.

2.Only one. This means the surface has a strong principal direction.

3.More than one. This means the surface has several directions.

Experimental results and the measured directions are shown in Figure 5.

\section{Illumination estimation}

Since the variances of mojette projections vary with the illumination tilt angle, we intuitively take this variance vector of a given image under an unknown tilt illumination as the feature vector to estimate its tilt illumination angle. Note that the $V M$ is nearly symmetrical in vertical, we only take six tilt angles for estimation $\left(0^{\circ}\right.$ to $150^{\circ}$ ). The detailed method is as follows.

All images in our experiments are of size $512 \times 512$. We divide all images to four quarters, and select two (top-left and top-right) for experiments. The top-left images compose our training set and the top-right images compose our testing set. The experiment is carried on within the surface image set.

For all training image sets, we calculate the $V M$ matrix. When given a test image from the corresponding test set, we calculate its variance vector $V$ of mojette projections. Then we compare the Euclidean distance between the feature vector $V$ and each column vector in $V M$. The estimated tilt angle is then assigned to the tilt angle of the image in the training set who has a minimum distance. Figure 4 shows the estimation accuracy of nine surface textures.

\section{Conclusion and future work}

In this paper, we propose a novel work in direction measurement and illumination estimation of 3D surface textures by using mojette transform. Variances of mojette projections on each image in a 3D surface set are utilized to form a matrix and then to measure the directionality the surface. Further more, this matrix is used to estimate the tilt illumination angle when given a sample image of a surface texture. Experiments show the efficiency and accuracy of our proposed method.

Future work can be done in improving the accuracy of the illumination estimation results and using whole tilt angles. Further more, the work of 3D surface texture classification with simultaneously illumination estimation can be investigated by using mojette transform. 


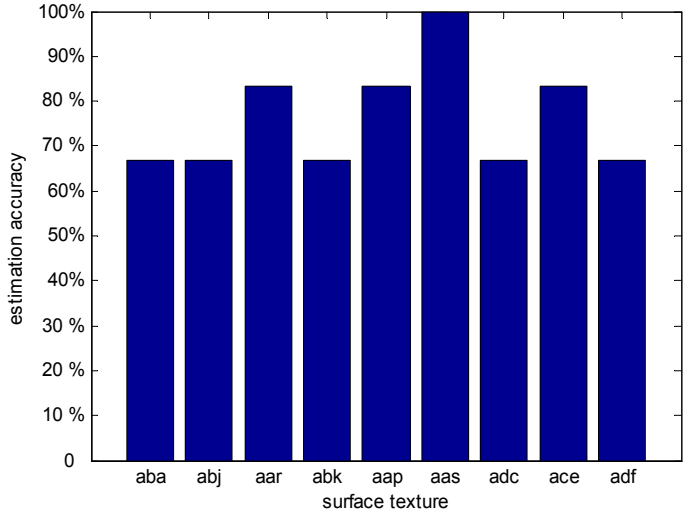

Figure 4. Tilt illumination angle estimation results

\section{References}

[1] PhoTex database. Texture lab, Heriot-Watt University, Edinburgh, UK. Available on-line at http://www.cee.hw.ac.uk/texturelab/database/photex/.

[2] F. Autrusseau, J. V. Guedon, and Y. Bizais. Mojette cryptomarking scheme for medical images. volume 5032, pages 958-965. SPIE, 2003.

[3] M. Chantler. The effect of illuminant direction on texture classification. $\mathrm{PhD}$ thesis, Dept. of Computing and Electrical Engineering, Heriot-Watt University, 1994.

[4] M. Chantler, M. Petrou, A. Penirsche, M. Schmidt, and G. McGunnigle. Classifying Surface Texture while Simultaneously Estimating Illumination Direction. International Journal of Computer Vision, 62(1):83-96, 2005.

[5] M. Chantler, M. Schmidt, M. Petrou, and G. McGunnigle. The effect of illuminant rotation on texture filters: Lissajous's ellipses. In ECCV '02: Proceedings of the 7th European Conference on Computer Vision-Part III, pages 289-303, London, UK, 2002. Springer-Verlag.

[6] J. P. Guédon, B. Parrein, and N. Normand. Internet distributed image information system. Integr. Comput.Aided Eng., 8(3):205-214, 2001.

[7] X. He, Y. Zhang, T. Lok, and M. Lyu. A New Feature of Uniformity of Image Texture Directions Coinciding with the Human Eyes Perception. Lecture Notes in Artificial Intelligence, Springer-Verlag, LNAI, 3614:727730, 2005.

[8] K. Jafari-Khouzani and H. Soltanian-Zadeh. Radon transform orientation estimation for rotation invariant texture analysis. IEEE Transactions on Pattern Analysis and Machine Intelligence, 27(6):1004-1008, 2005.

[9] J. RADON. Ober die Bestimmung von Funktionen durch ihre Integralwerte längs gewisser Mannigfaltigkeiten. Computed Tomography, 27, 1983.
[10] G. Vaidyanathan and P. Lynch. Texture direction analysis using edge counts. Southeastcon'89. Proceedings.'Energy and Information Technologies in the Southeast'., IEEE, pages 733-738, 1989.

[11] J. Wu and M. J. Chantler. Combining gradient and albedo data for rotation invariant classification of $3 \mathrm{~d}$ surface texture. In ICCV '03: Proceedings of the Ninth IEEE International Conference on Computer Vision, page 848 , Washington, DC, USA, 2003. IEEE Computer Society.

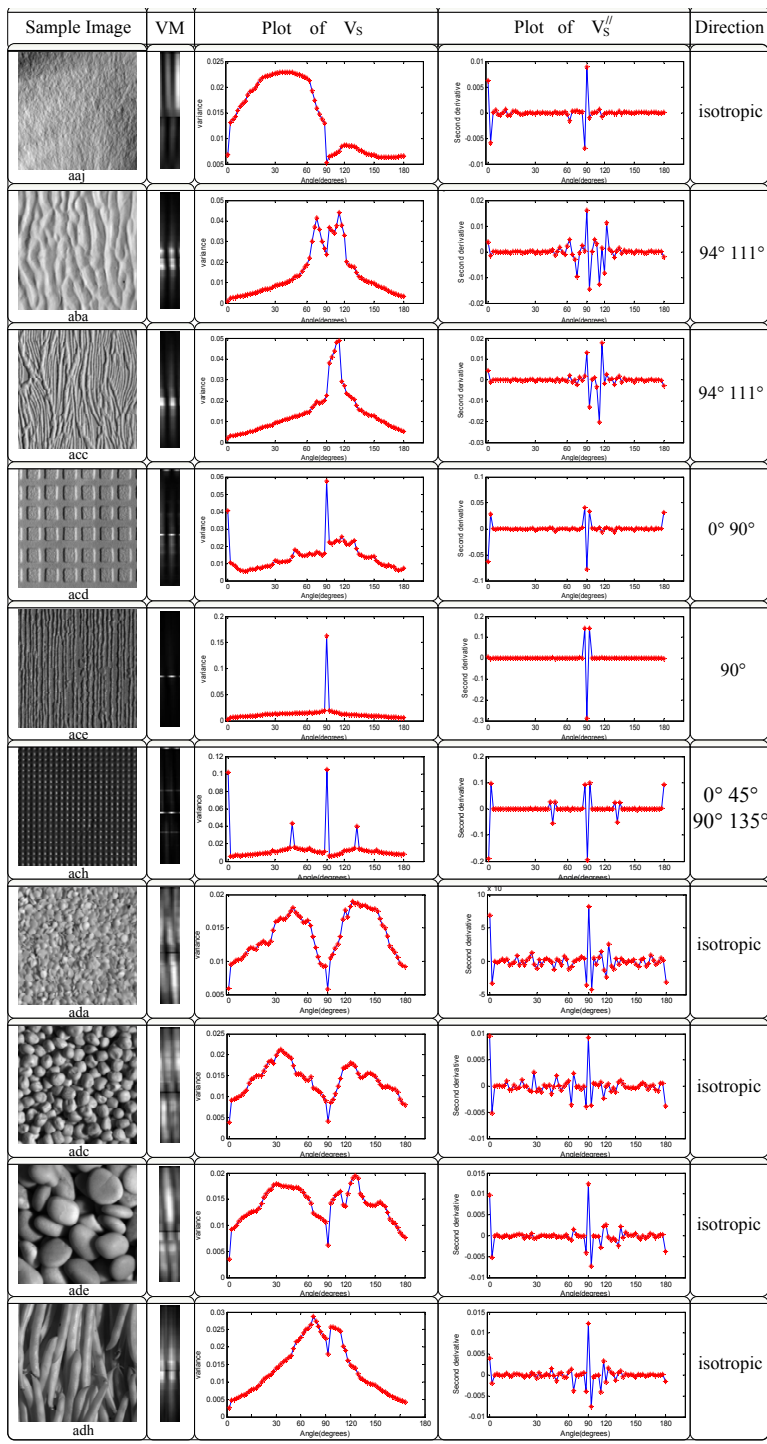

Figure 5. Direction measurement result. From left to right are sample image, image show of corresponding $V M$ matrix, plot of $V_{S}$, plot of $V_{S}^{\prime \prime}$ and the measured tilt angle. 